\title{
HRTEM techniques applied to nanocrystal modeling: towards an "atom-by-atom" description
}

\author{
D. G. Stroppa ${ }^{1,2}$, L. A. Montoro ${ }^{1}$, E. R. Leite $^{3}$ \& A. J. Ramirez ${ }^{1,2 *}$ \\ ${ }^{1}$ Brazilian Synchrotron Light Laboratory, Brazil \\ ${ }^{2}$ Mechanical Engineering School, University of Campinas, Brazil \\ ${ }^{3}$ Department of Chemistry, Federal University of São Carlos, Brazil
}

\begin{abstract}
The development of technologies based on nanostructures presents a wide range of challenges for materials scientists and engineers, including the attainment of well-controlled synthesis procedures, the improvement of characterization techniques down to the atomic scale resolution, and the conception and validation of reliable models that can describe materials properties as functions of their morphology and fabrication process. A relevant topic in this scenario is the correlation among the spatial distribution of chemical elements, the surface energy configuration, the growth mechanism, and the resultant nanocrystal 3D morphology.

This work presents an overview on the use of advanced HRTEM techniques for the quantitative analysis of nanocrystals and how these results can be used to implement nanocrystals models, which can analytically describe the material features on an atomic level. The presented findings show the combined use of experimental data and theoretical tools, such as image simulation and ab initio surface energy calculations, for the advanced quantitative characterization of nanocrystalline systems.

The combination of experimental and theoretical efforts on HRTEM characterization represents a powerful tool for the nanocrystal 3D morphology elucidation with atomic resolution and the chemical/structural properties assessment in a quantitative way. Thereby, it is presented as the stepping stone towards the development of novel approaches to describe nanostructured systems.
\end{abstract}

Keywords: HRTEM, nanocrystals modeling, quantitative analysis. 


\section{Introduction}

Nanotechnology has been considered one of the most promising branches on the scientific development since the 60's [1] and its contributions to various fields are notorious nowadays. The "nano" suffix and its effects became an intrinsic subject of materials science and technology on the last few years as the unique properties from nanometer scale are becoming increasingly relevant in those fields.

The traditional materials engineering perspective [2], depicted in Figure 1, can also be applied for the analysis of nanostructured systems. This approach considers that the design of materials aiming an optimum performance requires the complete understanding of the correlation among the processing routes parameters, the materials structure and the resultant properties. However, regarding nanostructured systems, this correlation may be stated as the interrelationship among the synthesis parameters, the atomic scale morphology and the energy configuration [3]. As the surface area to volume ratio is enhanced for such systems, unique properties related to the surface energy distribution become relevant. These properties are the source for the unique performance of nanomaterials [4].

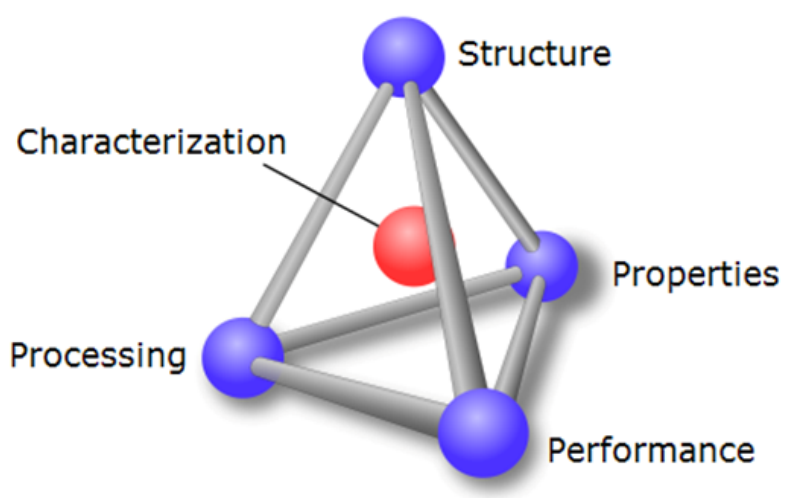

Figure 1: Engineering approach for describing and correlating materials features.

Materials characterization plays a crucial role on the development of reliable models for the description and the design of nanostructured materials with specific features for countless technological areas. Apart from the specific questions that different characterization techniques may answer, there are three general issues that can be highlighted as the most relevant questions for the nanostructured materials modeling and design.

The first one is related to the morphology characterization at the atomic scale. As mentioned before, it is vital to assess the nanomaterials structure with high resolution in order to evaluate its correlation with the system properties. The ultimate goal in this sense is the "atom-by-atom" description of nanocrystals, 
which would be specially required for the determination of atomic species segregation on alloyed/doped materials [5] and the high resolution strain state analysis of nanostructures [6].

The second issue is related to the nanocrystals surface structure and its dependency on the synthesis environment and chemical species. This aspect is intrinsically correlated to the nanocrystals morphology and, consequently, to the material surface energy distribution and properties [7].

The third relevant point is the interrelation between the nanocrystals and the system growth behavior [8]. These aspects represent crucial issues for the understanding and optimization of synthesis methodology as they are closely related to the nanostructured system stability and long term reliability [9].

As describing the properties and behavior of each nanocrystal in an actual nanostructured system represents an overwhelming many-bodies problem, simplified models are needed for the engineering of nanomaterials in a reliable manner. Nanocrystal modeling [10] is a wide approach where nanostructured systems components are described as building blocks with specific morphology, energy distribution, and interaction mechanisms. In these terms, the development of accurate models may be a fundamental tool for nanocrystals and mesocrystals shapes prediction, design and control of growth processes, and the resulting properties tuning of an unlimited number of systems.

The development of reliable modeling methodologies represents a hard task for materials scientists and engineers due to involved challenges, especially when quantitative high resolution characterization analyses are required. Even though advanced characterization techniques based on electron microscopy, scanning probe microscopy and synchrotron radiation are feasible and complimentary in this scenario, limitations [11] associated to each one of them prevent their isolated use a for reliable analysis. The most relevant limitations of high resolution techniques are summarized on Table 1.

Table 1: Most relevant limitations from high resolution characterization tools.

\begin{tabular}{cc}
\hline ISSUE & EFFECT \\
\hline Sample Preparation & modification on the sample original features \\
Sample Stability & characteristics changes during analysis \\
Energy Resolution & spectroscopic signature may have overlaps \\
Signal to Noise Ratio & poor detection system sensitivity \\
Sampling & lack of statistical representativeness \\
Averaging & lack of features from individual particles \\
Data Analysis & deconvolution, modeling and fitting \\
\hline
\end{tabular}

Although continuous improvements on the characterization instrumentation and methodologies enhance the individual techniques performance, the combined use different available experimental techniques and theoretical approaches are widely used to overcome the inherent limitations of the quantitative analysis of nanosized systems. 
Transmission Electron Microscopy (TEM) based techniques stand out among other characterization techniques for nanostructured systems due to the possibility of a comprehensive and versatile approach including high resolution imaging, diffraction and spectroscopy. This multipurpose characteristic allied with simulation procedures provides complementary information that can be used to improve the analysis precision.

Recent developments on the TEM instrumentation and analysis methods allowed outstanding advances on nanoscience and nanotechnology [12]. The spherical aberration (Cs) correction [13] can be considered among the most influential improvements on both high resolution TEM (HRTEM) and high resolution scanning TEM (HRSTEM) due to a number of factors. For HRTEM, the Cs-correction of the objective lens resulted in the drastic improvement on achievable spatial resolution, the possibility of larger gaps in the pole piece for in situ experiments, and new imaging modes [14] due to fine tuning of the aberration coefficients. For HRSTEM, the Cs-correction of the condenser lens leads to an improvement on the achievable spatial resolution due to the probe size reduction and the effective beam current increase.

In addition to the instrumental TEM improvements, the development of more accurate models to describe the image formation and to support the TEM image simulation [15] provides a more reliable interpretation of experimental data.

This work presents an overview on the HRTEM techniques state of art with several examples which indicate the development of such characterization tools towards the quantitative high resolution analysis of nanocrystalline materials.

\section{HRTEM and HRTEM image simulation}

HRTEM image formation is based on the incident electron beam scattering by a thin sample. The technique is especially relevant for very thin crystalline samples, where the interaction of the several diffracted beams forms an interference patterns. As the diffracted components correspond to particular oriented periodic spacings on the sample, their interference may appear as a $2 \mathrm{D}$ periodic image which cannot be directly interpreted. The direct interpretation unfeasibility is mainly related to the HRTEM interference pattern dependency with the sample thickness and with the microscope configuration, especially the defocus value. However, the use of HRTEM image simulation can provide information about the sample crystalline arrangement.

HRTEM image simulation is a crucial step in HRTEM characterization, which supports the atomic structure analysis. Multislice method [16] is the most widely used image simulation procedure and consists in the electrons wave function calculation after the electron beam interaction with the sample projected potential. Imaging distortions due to the lens aberrations, microscope instabilities and detection system defects [17] may be included in order to better reproduce the experimental imaging condition.

The HRTEM imaging and image simulation combination has provided numerous examples of successful materials characterization. Remarkable examples can be noticed on the determination and refinement of crystalline 
structures [18] and the quantitative evaluation of the chemical composition profile along the thin films interface [19].

Figure 2 illustrates the combination of HRTEM imaging and image simulation on the evaluation of an anomalous anisotropic growth mechanism for $\mathrm{SnO}_{2}$ nanocrystals [20]. The used approach provided an unambiguous characterization of the growth mechanism and its preferential direction, which would not be feasible from direct imaging only.
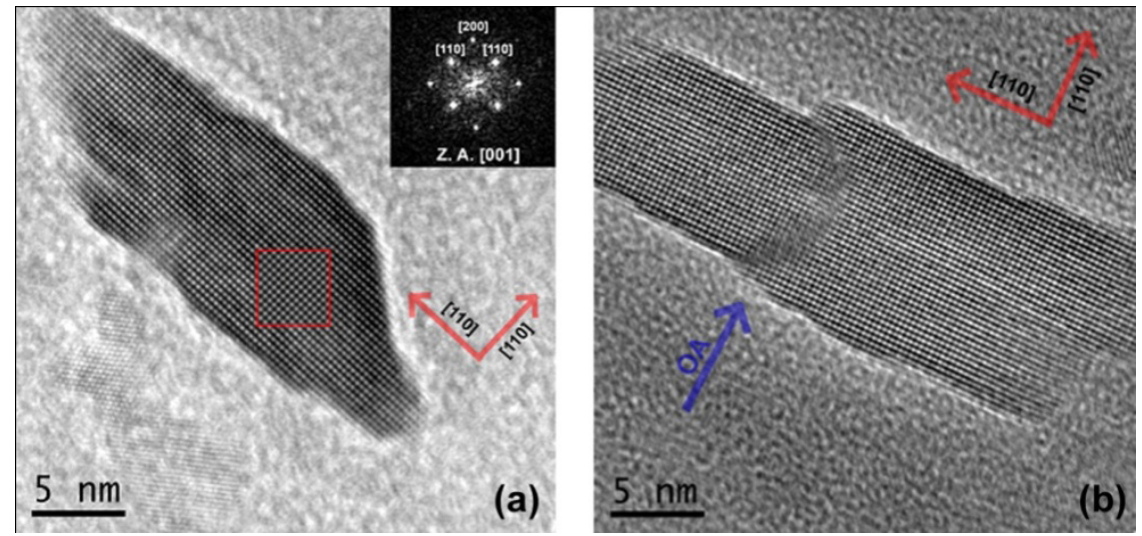

Figure 2: (a) $\mathrm{SnO}_{2}$ nanocrystal HRTEM image with an indexed FFT inset and a HRTEM multislice simulation (red square). (b) oriented attachment along the [110] is identified as the main growth mechanism. [From ref. [20], copyright (C) 2011 by RSC Publishing, reprinted with permission of authors.]

In addition, the accurate determination of nanocrystals 3D morphology by HRTEM simulation of nanocrystals [21] can be used to indirectly extract quantitative dopant segregation information [22] from systems where conventional analytical techniques are impracticable due to several experimental restrictions. Figure 3 depicts the 3D morphological modeling for $\mathrm{Sb}: \mathrm{SnO}_{2}$ nanocrystals with different dopant contents. The nanocrystals models were compared to the Wulff [23] constructions based on ab initio surface energy calculations, providing the dopant atoms segregation for individual particles and its dependency with the doping level [24].

The presented examples depict the synergism between the HRTEM imaging and theoretical methods such as HRTEM image simulation and ab initio surface energy calculations. Therefore, this combination presents a remarkable potential as a tool for unveiling important nanocrystalline systems features, such as 3D morphology, dopant segregation, surface energy distribution, and growth mechanisms. 


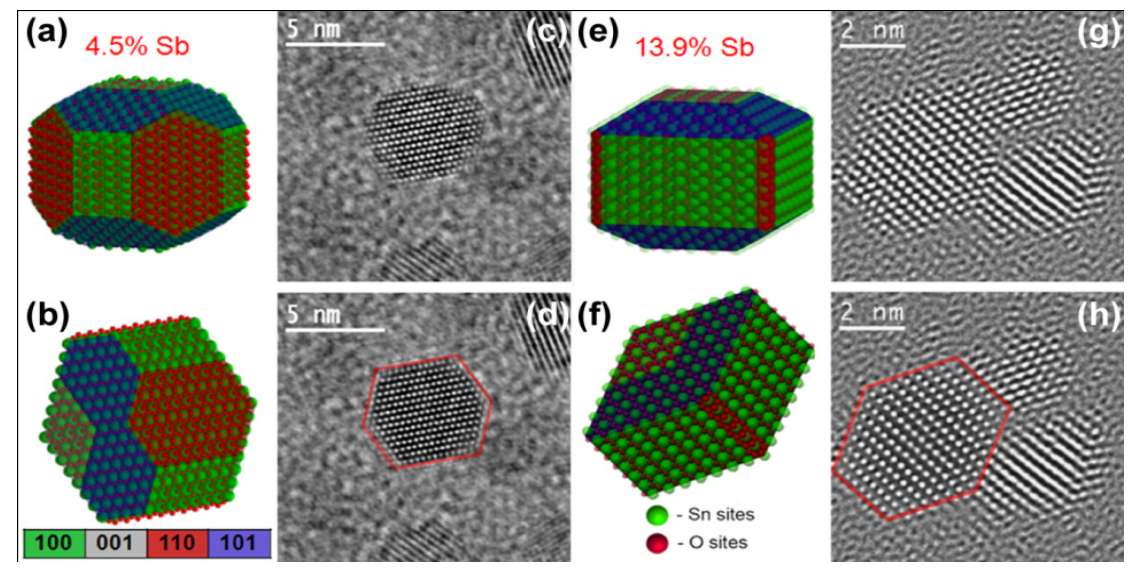

Figure 3: (a), (e) Trimetric and (b), (f) [111] zone-axis oriented views for the Sb-doped nanocrystals geometric model. (c), (g) Original HRTEM images and (d), (h) superimpositions with the simulated HRTEM images. [Adapted from ref. [22], copyright (C) 2009 by ACS Publications, reprinted with permission of authors.]

\section{Focal series reconstruction and geometric phase analysis}

Although the HRTEM image simulation can support accurate nanocrystals 3D morphology analyses, it is not always feasible to use such procedure to precisely evaluate the position of individual atoms or atomic columns in crystalline structures. To extract information which is directly related to the atomic positions, such as strain/stress state, a refinement on the HRTEM analysis is needed.

Focal Series Reconstruction (FSR) [25] is a proposed calculation which is applied for the electron wavefunction restoration on the HRTEM imaging process and allows obtaining the sample projected potential without the interference of the microscope optical aberrations. The FSR implementation is based on the acquisition of a set of images under different objective lens defocus conditions, which is subsequently submitted to a restoration procedure that estimates the contrast transfer function (CTF) and the aberration coefficients implicated on the imaging process.

A number of successful uses of FSR aiming a straightforward image interpretation [26], the correction of residual aberration [27], and the improvement of the HRTEM technique spatial resolution [28] have been already described in the literature. An example which illustrates the image improvement when FSR method is applied to structural analysis is depicted on Figure 4. These images show the comparison between an original HRTEM image and a phase image of the reconstructed wavefunction [29].

Geometric Phase Analysis (GPA) [30] is a methodology for measuring and mapping structural displacement fields on HRTEM images using a reference lattice. Its application to HRTEM image analysis allows the local lattice distortion evaluation, which can be directly related to the local strain state. This 
method has been effectively employed to study strain fields in semiconductors and metals [31].

The GPA implementation is based on the reciprocal space evaluation of a HRTEM image with respect to a reference undistorted lattice image, either obtained from a different region in the analyzed sample or from an image simulation procedure. As a spatial frequency spread around the characteristic lattice parameters frequencies is existent on strained samples, the determination of lattice distortions can be performed by the comparison with a reference image. The distorted components can be extracted on the reciprocal space and further translated to real space components through an inverse Fourier transform. Such analysis can be performed for two non-collinear spatial frequencies, resulting in a projected $2 \mathrm{D}$ distortion description of the sample which can be related to its $2 \mathrm{D}$ strain/stress state.
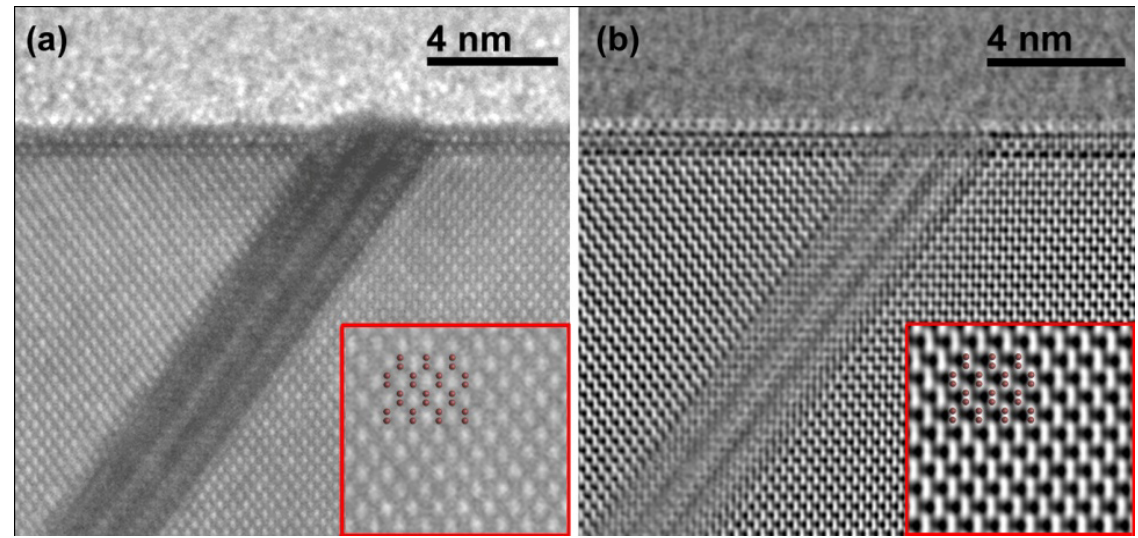

Figure 4: (a) Example of an original HRTEM image and (b) the output from a FSR restoration from a $\mathrm{CoSi}_{2}$ sample. The insets exhibit a higher magnification detail with the crystal structure superimposition. [Adapted from ref. [29], reprinted with permission of authors.]

The GPA application is reported on the literature as an efficient tool to calculate strain maps on microelectronic components [32] and for the strain state analysis on individual nanocrystals [33]. A typical output from a GPA analysis is the distortion map from the analyzed HRTEM image, as exemplified on Figure 5 , which includes the perpendicular, parallel and rotational strain components.

An example of the combined user of FSR and GPA is presented on Figure 6 [34]. Cross-section HRTEM images of epitaxially grown $\mathrm{Si}_{1-\mathrm{x}} \mathrm{Ge}_{\mathrm{x}}: \mathrm{Si}(001)$ alloyed islands were used to characterize in a self-consistent way the strain configuration and the local chemical composition with high spatial resolution. By the combination of projected 2D chemical composition mapping from two different cross-sectioned zone-axis views, [100] and [110], it was possible to infer the chemical composition in a three-dimensional fashion [35]. 

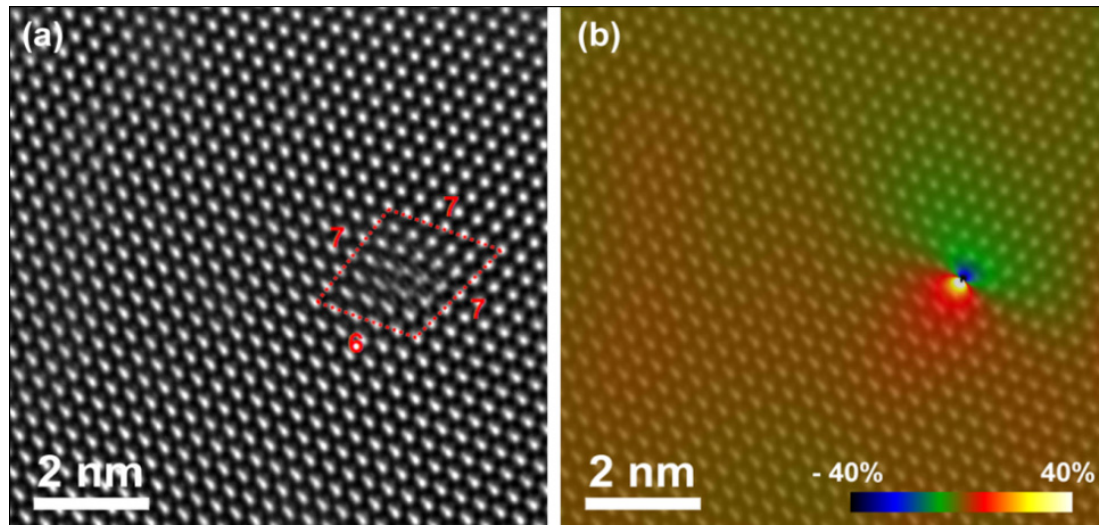

Figure 5: (a) Original Cs-corrected HRTEM image and (b) distortion mapping from a single dislocation on $\mathrm{CeO}_{2}$ sample.

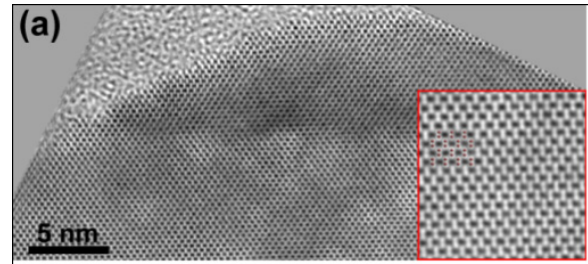

(b)
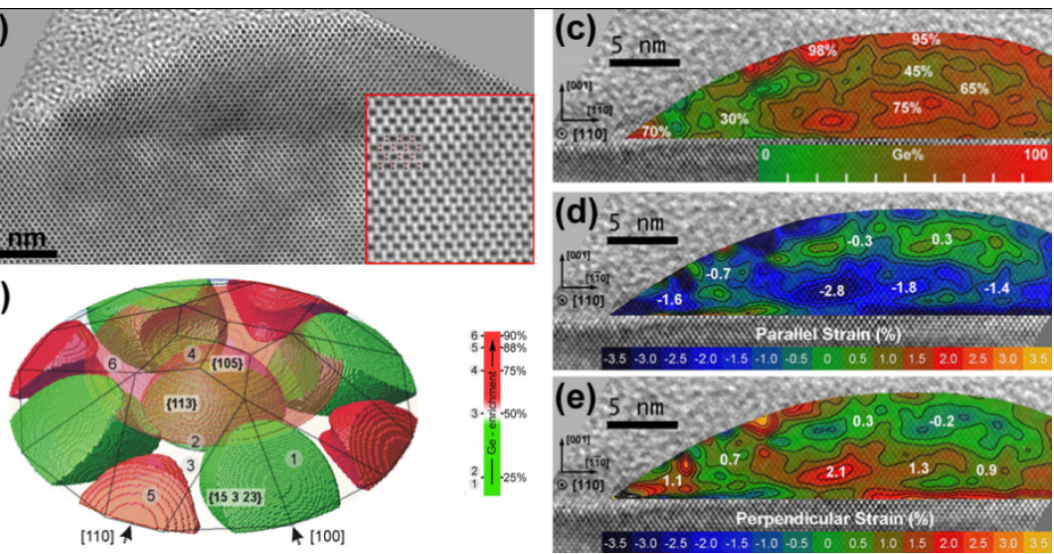

Figure 6: (a) FSR reconstruction from a Si-Ge island including a higher magnification detail on the inset. (b) A simplified 3D chemical reconstruction was obtained after the strain chemical mapping of several Si-Ge islands at different zone-axis. An example from a single island: (c) chemical composition map, (d) parallel and (e) perpendicular strain projections. [Adapted from refs. [34] and [35], copyright (C) 2009 by ACS Publications, reprinted with permission of authors.]

The successful application of this technique to quantify the elastic behavior of the $\mathrm{Si}-\mathrm{Ge}: \mathrm{Si}(001)$ system shows that this methodology arises as a remarkable tool for accurate chemical and elastic state evaluation, which can be applied to several strained alloyed nanostructures, such as epitaxial islands, nanowires, nanocrystals, and thin films. 


\section{A novel approach for HRTEM imaging}

The improvement of HRTEM characterization depends on the comprehensive understanding of the underlying image formation theory. An important imaging condition representation is given by the Contrast Transfer Function (CTF), which represents how the spatial frequencies are transmitted through microscope imaging system.

Since the 40's, microscopists have been trying to improve the CTF characteristics by tuning the instruments parameters during the experiments or by the posterior treatment of the images. The main goal in this sense is to extend information transfer for high spatial frequencies. The most remarkable improvements were achieved by using induced defocus values on the objective lens in order to compensate its inherent positive spherical aberration coefficient [36]. However, a ground-breaking evolution happened on HRTEM with the Cscorrection possibilities.

At a first glance, the use of Cs-correction adds an additional degree of freedom to the microscope tuning, allowing the CTF direct improvement for high spatial frequencies and improving the microscope information limit. A comparison between the HRTEM images from the same sample obtained by conventional and Cs-corrected microscopes, which is presented on Figure 7, clearly shows the image enhancement provided by the aberration corrector hardware.

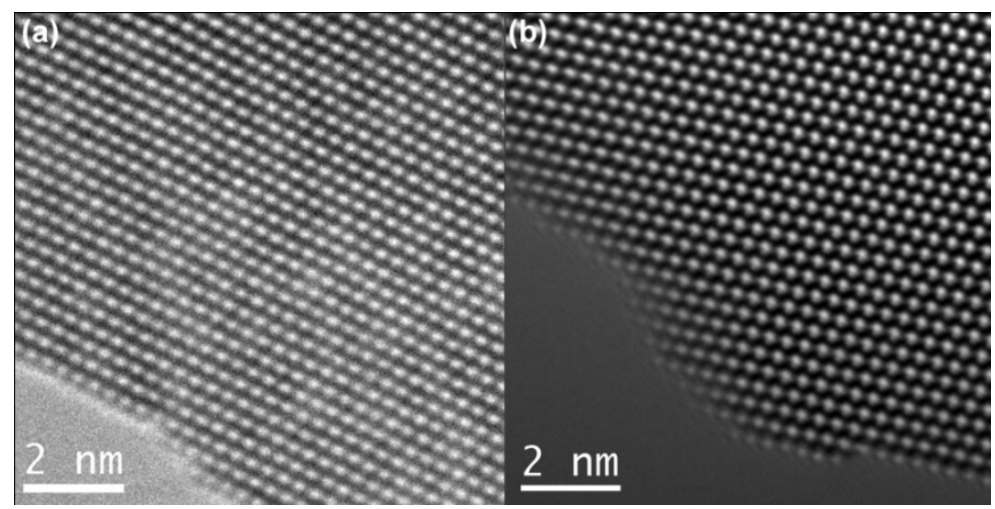

Figure 7: $\quad$ Raw HRTEM images from the same $\mathrm{CeO}_{2}$ nanocrystal from a (a) standard TEM microscope (1.25 $\AA$ information limit) and from a (b) Cs-corrected microscope $(0.8 \AA$ information limit).

A detailed investigation [37] of the electron scattering on the dynamical regime pointed out that non-linear contributions would enable the achievement of enhanced contrast transfer for low-Z elements by the use of negative Cs values. The Negative Cs Imaging (NCSI) [14] aims the contrast maximization at high frequencies by the microscope parameters optimization, including defocus and Cs adjustments. The NCSI application allowed some unique measurements 
including the high resolution assessment of light elements atomic columns information, such as occupancy next to crystalline defects [38] and sub-angstrom displacement in magnetic domain walls [39].

Examples of NCSI HRTEM characterization for $\mathrm{CeO}_{2}$ sintered and nanocrystalline samples are depicted on Figure 8. Although the contrast for Oxygen columns is observed in both images, the direct analysis of a bulk-like sample HRTEM image (Figure 8a) is unfeasible due to a combination of factors. As the accurate thickness estimation is not achievable by HRTEM image simulation for this specific combination of crystalline system and imaging conditions, the intensity distribution cannot be directly associated to the Oxygen columns scattering. A solution for this issue is the sample projected potential assessment by the FSR application on NCSI HRTEM images (Figure 8b).
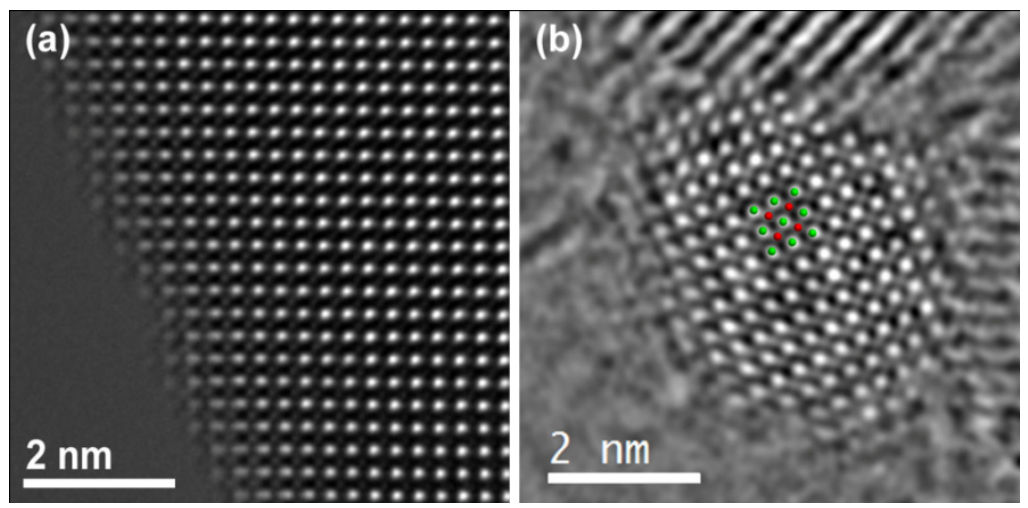

Figure 8: (a) NCSI HRTEM image from a $\mathrm{CeO}_{2}$ bulk-like sample on the [100] ZA and (b) FSR from NCSI HRTEM images from a $\mathrm{CeO}_{2}$ nanocrystal on the same $\mathrm{ZA}$ orientation including a unit cell superimposition.

\section{Concluding remarks}

State of art HRTEM techniques indicate that their use in conjunction with theoretical procedures for HRTEM image simulation and data analysis represents a vital tool for the quantitative evaluation of nanostructured systems. The depicted examples illustrate how this technique can simplify, or even present completely novel approaches, a number of materials characterization challenges where a quantitative high resolution assessment is required.

The current development of HRTEM instrumentation and related methodologies indicates that its importance on materials characterization will grow on this decade, especially due to the novel possibilities achieved by aberration correction implementation and by on-growing combined use with HRSTEM imaging and spectroscopy techniques. 


\section{Acknowledgements}

The authors acknowledge the financial support of the Brazilian research funding agencies FAPESP and FINEP; the German research funding agency DAAD. The authors would also like to thank the Ernst Ruska Centre staff at the Forschungszentrum Jülich and the QFA staff at the Universitat Jaume I for the fruitful discussions and scientific support.

\section{References}

[1] Feynman, R. P.; There's plenty of room at the bottom; Eng. Sci., 23(5), 22-36, 1960.

[2] Callister, W. D.; Fundamentals of Materials Science and Engineering; John Wiley \& Sons: New York, pp. 2-5, 2001.

[3] Burda, C.; Chen, X.; Narayanan, R.; El-Sayed, M. A.; Chemistry and properties of nanocrystals of different shapes; Chem. Rev., 105(4), 1025-1102, 2005.

[4] Kolmakov, A.; Moskovits, M.; Chemical Sensing and Catalysis by OneDimensional Metal-Oxide Nanostructures; Annu. Rev. Mater. Res.; 34, 151-180, 2004.

[5] Shibata, N.; Pennycook, S. J.; Gosnell, T. R.; Painter, G. S.; Shelton, W. A.; Becher, P. F.; Observation of rare-earth segregation in silicon nitride ceramics at subnanometre dimensions; Nature; 428, 730-733, 2004.

[6] Brune, H.; Giovannini, M.; Bromann, K.; Kern, K.; Self-organized growth of nanostructure arrays on strain-relief patterns; Nature, 394, 451-453, 1998.

[7] Yang, H. G.; Sun, C. H.; Qiao, S. Z.; Zou, J.; Liu, G.; Smith, S. C.; Cheng, H. M.; Lu, G. Q.; Anatase TiO2 single crystals with a large percentage of reactive facets, Nature, 453, 638-641, 2008.

[8] Dalmaschio, C. J.; Ribeiro, C.; Leite, E. R.; Impact of the colloidal state on the oriented attachment growth mechanism; Nanoscale, 2, 2336-2345, 2010.

[9] Yu, W. W.; Wang, Y. A.; Peng, X.; Formation and Stability of CdTe Nanocrystals; Chem. Mater.; 15, 4300-4308, 2003.

[10] Barnard, A. S.; Modelling of Nanoparticles: Approaches to Morphology and Evolution; Rep. Prog. Phys., 73, 1-52, 2010.

[11] Brundle, C. R.; Evans, C. A.; Wilson, S.; Encyclopedia of Materials Characterization, Butterworth-Heinemann, Reed Publishing USA, 1992.

[12] Hawes, P.; Advances in Imaging and Electron Physics: Aberrationcorrected Electron Microscopy; Elsevier, 153, 2008.

[13] Haider, M.; Uhlemann, S.; Schwan, E.; Rose, H.; Kabius, B.; Urban, K.; Electron Microscopy Image Enhanced; Nature, 392, 768-769, 1998.

[14] Jia, C. L.; Lentzen, M.; Urban, K.; High-resolution transmission electron microscopy using negative spherical aberration; Microscopy and Microanalysis, 10(2), 174-184, 2004. 
[15] Stadelmann, P. A.; EMS - A Software Package for Electron Diffraction Analysis and HREM Image Simulation in Materials Science; Ultramicroscopy, 21(2), 131-145, 1987.

[16] Cowley, J. M.; Moodie, A. F.; The Scattering of Electrons by Atoms and Crystal; Acta Crystallographica, 10(10), 609-619, 1957.

[17] Thust, A.; High-Resolution Transmission Electron Microscopy on an Absolute Contrast Scale; Physical Review Letters, 102(22), 1-4, 2009.

[18] Kilaas, R.; Radmilovic, V.; Structure determination and structure refinement of $\mathrm{Al}_{2} \mathrm{CuMg}$ precipitates by quantitative high-resolution electron microscopy; Ultramicroscopy, 88(1), 63-72, 2001.

[19] Tillmann, K.; Luysberg, M.; Specht, P.; Weber, E. R.; Direct compositional analysis of AlGaAs/GaAs heterostructures; Ultramicroscopy, 93, 123-137, 2002.

[20] Stroppa, D. G.; Montoro, L. A.; Beltran, A.; Conti, T. G.; Silva, R. O.; Andres, J.; Leite, E. R.; Ramirez, A. J.; Anomalous oriented attachment growth behavior on $\mathrm{SnO}_{2}$ nanocrystals; Chem. Commun.; 47, 3117-3119, 2011.

[21] Stroppa, D. G.; Montoro, L. A.; Righetto, R. D.; Ramirez, A. J.; MEGACELL: A nanocrystals models construction software for HRTEM multislice simulation; Ultramicroscopy; 2011, doi:10.1016/ j.ultramic.2011.03.013.

[22] Stroppa, D. G.; Montoro, L. A.; Beltran, A.; Conti, T. G.; Silva, R. O.; Andres, J.; Longo, E.; Leite, E. R.; Ramirez, A. J.; Unveiling the Chemical and Morphological Features of Sb:SnO $\mathrm{O}_{2}$ Nanocrystals by the Combined Use of HRTEM and ab initio Surface Energy Calculations; J. Am. Chem. Soc.; 131, 14544-14548, 2009.

[23] Herring, C.; Some Theorems on the Free Energies of Crystal Surfaces; Phys. Rev.; 82(1), 87-93, 1951.

[24] Stroppa, D. G.; Montoro, L. A.; Beltran, A.; Conti, T. G.; Silva, R. O.; Andres, J.; Leite, E. R.; Ramirez, A. J.; Dopants segregation analysis on $\mathrm{Sb}: \mathrm{SnO}_{2}$ nanocrystals; submitted for publication, 2011.

[25] Thust, A.; Coene, W. M. J.; de Beeck, M. O.; Van Dyck, D.; Maximumlikelihood method for focus-variation image reconstruction in high resolution transmission electron microscopy; Ultramicroscopy, 64(1), 211-230, 1996.

[26] Barthel J.; Weirich T. E.; Cox, G.; Hibst, H.; Thust, A.; Structure of $\mathrm{Cs}_{0.5}\left[\mathrm{Nb}_{2.5} \mathrm{~W}_{2.5} \mathrm{O}_{14}\right]$ analysed by focal-series reconstruction and crystallographic image processing; Acta Materialia, 58(10), 3764-3772, 2010.

[27] Kirkland A. I.; Meyer, R. R.; "Indirect" high-resolution transmission electron microscopy: Aberration measurement and wavefunction reconstruction; Microscopy and Microanalysis; 10(4), 401-413, 2004.

[28] Biggemann, D.; da Silva, M. H. P.; Rossi, A. M.; Ramirez, A. J.; High-Resolution Transmission Electron Microscopy Study of Nanostructured Hydroxyapatite; Microscopy and Microanalysis, 14, 433438, 2008. 
[29] Montoro, L. A. et al; unpublished data.

[30] Kret, S.; Ruterana, P.; Rosenauer, A.; Gerthsen, D.; Extracting quantitative information from high resolution electron microscopy; Phys. Stat. Sol., 227(1), 247-295, 2001.

[31] Hytch, M. J.; Putaux, J. M.; Penisson, J. M. ; Measurement of the displacement field of dislocations to 0.03 angstrom by electron microscopy; Nature, 423, 270-273, 2003.

[32] Hue, F.; Hytch, M.; Bender, H.; Houdellier, F.; Claverie, A.; Direct mapping of strain in a strained silicon transistor by high-resolution electron microscopy; Phys. Rev. Lett., 100(15), 1-4, 2008.

[33] Johnson, C. L.; Snoeck, E.; Ezcurdia, M.; Rodriguez-Gonzalez, B.; Pastoriza-Santos, I.; Liz Marzan, L. M.; Hytch, M. J.; Effects of elastic anisotropy on strain distributions in decahedral gold nanoparticles; Nature Materials, 7, 120-124, 2008.

[34] Montoro, L. A.; Leite, M. S.; Biggemann, D.; Peternella, F. G.; Batenburg, K. J.; Medeiros-Ribeiro, G.; Ramirez, A. J.; Revealing Quantitative 3D Chemical Arrangement on Ge-Si Nanostructures; J. Phys. Chem. C., 113(21), 9018-9022, 2009.

[35] Montoro, L. A.; Medeiros-Ribeiro, G.; Ramirez, A. J.; Novel Approach for High-Resolution Elastic Behavior Assessment of Alloyed Strained Nanostructures; J. Phys. Chem. C., 114(29), 12409-12415, 2010.

[36] Scherzer, O.; The Theoretical Resolution Limit of the Electron Microscope; J. Appl. Phys., 20(1), 20-29, 1949.

[37] Jia, C. L.; Houben, L.; Thust, A.; Barthel, J.; On the benefit of the negative-spherical-aberration imaging technique for quantitative HRTEM; Ultramicroscopy, 110(5), 500-505, 2010.

[38] Jia, C. L.; Lentzen, M.; Urban, K.; Atomic-resolution imaging of oxygen in perovskite ceramics; Science, 299, 870-873, 2003.

[39] Jia, C. L.; Mi, S. B.; Urban, K.; Vrejoiu, I.; Alexe, M.; Hesse, D.; Atomic-scale study of electric dipoles near charged and uncharged domain walls in ferroelectric films; Nature Materials, 7, 57-61, 2008. 